\title{
The kinetics research on coal tar pitch and its extraction
}

\author{
Chuanli $\mathrm{Xu}^{\mathrm{a}}$, Xianglin Cheng ${ }^{\mathrm{b} *}$, Liucheng $\mathrm{Wang}^{\mathrm{c}}$, Jianhong Zhao ${ }^{\mathrm{d}}$, Jianshe \\ Wang $^{\mathrm{e}}$, Chengying Song ${ }^{\dagger}$,
}

\author{
Zhengzhou University, School of Chemical Engineering and Energy \\ Resources and Materials Collaborative Innovation Center of He'nan Province

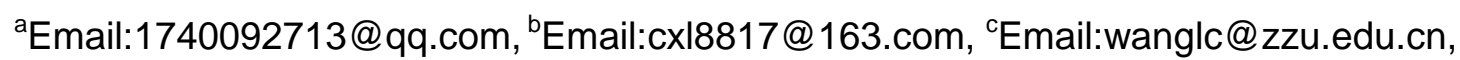 \\ dEmail:zhaojh@zzu.edu.cn, ${ }^{\text {EEmail:wangjs@zzu.edu.cn, }{ }^{\mathrm{f}} E m a i l: s o n g c y @ z z u . e d u . c n}$
}

Keyword: Coal tar pitch; thermogravimetric analysis; kinetics

Abstract: The kinetics of pyrolysis characteristics of coal tar pitch (CTP) and quinoline soluble of CTP (refined pitch) were investigated by thermogravimetric analysis at the heating rate of $5,10,15$, $20^{\circ} \mathrm{C} / \mathrm{min}$, respectively. The reaction kinetics parametres were obtained based on Kissinger method, Flynn-Wall-Ozawa method and Friedman method, respectively. The results show that pyrolysis process of CTP and the refined pitch includes three stages. Different heating rate has different effect on carbonization. The pre-exponential factor $\ln$ A of CTP and the refined pitch is 14.40 and 12.06, respectively. The reaction mechanism function of CTP and the refined pitch is $[-\ln (1-\alpha)]$. The CTP shows more significant temperature sensitivity and higher reactivity than the refined pitch.

\section{Introduction}

China has the largest coal coke output in the world, which is about 477 million tons in 2014. The annual output of coal tar in coking is more than 22 million tons. Coal tar pitch (CTP), by-product of coal tar distillation, occupies $54-60 \%$ of the amount of coal tar [1]. CTP is used as materials to prepare not only linoleum, buildings waterproof layer, high-grade pitch paint, preparing pitch coke, delayed coke and modified asphalt material etc, but also carbon electrode, anode aluminum electrolytic binder, battery production and refractory adhesive. But the existing utilization methods have many defects such as low added value, serious pollution, and small market capacity. The coal coking industry are faced with the common problems on finding the way to process CTP until now. CTP contains a large number of high quality aromatic hydrocarbon, so it is an urgent problem to develop high added value processing method. And deep processing is also the traditional coal coking industry upgrading and transformation needs in China.

CTP is a very complex system, containing a large number of aromatic ring organic compounds. It is estimated that there are more than 10000 different chemical compositions in CTP [2]. A large number of aromatic components are in the form of 2-6 ring condensation of aromatic rings. CTP is able to be used as raw materials for high performance carbon materials, like needle coke, mesophase pitch based carbon fiber, carbon foam, impregnant pitch, mesocarbon microbeads, etc. However, there is no mature raw material refining process in China, resulting in long-term slow progress in industrialization of high performance carbon materials and other high value-added product due to lack of mastering the basic characteristics of CTP. Thermal analysis is a certain kind of testing technology for studying the physical and chemical changes in the process of heating and cooling. By thermal analysis method, the reaction kinetics can be effectively studied, and the kinetic parameters, such as thermal reaction series, reaction rate and activation energy, are also determined. 
Thermal analysis had applied to the research of CTP in order to obtain the thermal reactivity of CTP[3-5].

In this study, synchronous thermal analysis (including Kisssinger method, Flynn- Wall-Ozawa method and Friedman method) is used to analyze the dynamic parameters of CTP and the refined pitch, The result gives some clue on how to develop a new technology on refining, modification and deep processing of pitch.

\section{Experimental}

CTP is selected from Jiyuan Jinma Coking Co. ltd.. Quinoline soluble of CTP is the refined pitch. Its element analysis is listed in table 1.

Table 1 Elemental analysis of CTP

\begin{tabular}{lrrlll}
\hline Sample & $\mathrm{C} \%$ & $\mathrm{H} \%$ & $\mathrm{~S} \%$ & $\mathrm{~N} \%$ & $\mathrm{H} / \mathrm{C}$ \\
\hline CTP & 89.89 & 4.528 & 0.850 & 1.61 & 0.604 \\
Refined pitch & 90.49 & 4.888 & 0.911 & 1.46 & 0.648 \\
\hline
\end{tabular}

The element analysis is carried out on EL-2 from German Vario company. Thermal analysis is executed on TG-DSC60 (Shimadzu Corporation).

The sample, weighted $10 \pm 0.5 \mathrm{mg}$, were heated from room temperature up to $800^{\circ} \mathrm{C}$ with $5,10,15$, $20^{\circ} \mathrm{C} / \mathrm{min}$, under the atmosphere of N2 (99.999\%), TG-DTG curves are then attained, respectively.

\section{Results and discussion}

\section{Pyrolysis characteristics}

The TG/DTG curves of CTP and the refined pitch are listed in Fig. 1and Fig. 2, respectively. TG/DTG curve of CTP and the refined pitch are shown similar trend. With different heating rates, both pitch show a similar weight loss trend, and the weight loss rate increase at first and then decrease. However, with the increase of heating rate, the overall heating curves display right shift and the maximum weight loss rate decreases, which is due to the shorter reaction time of the two pitches. But the curves of CTP and the refined pitch are different at the amount of left carbonized solid residue. The high to low order of CTP is $10,15,20,5^{\circ} \mathrm{C} / \mathrm{min}$, while the refined pitch is 5,10 , $15,20{ }^{\circ} \mathrm{C} / \mathrm{min}$. The difference lies in the different component of the two pitches. The refined pitch is attained through removal of macromolecular aromatic ring compounds by solvent extraction of CTP. The molecular weight distribution of the refined pitch is narrower and smaller than that of CTP. There is no macromolecular aromatic ring compounds in the refined pitch. Slower rising temperature would induce the less volatile from the refined pitch, leading to higher amount of carbonized solid residue. 


\section{Kinetics analysis on CTP and the refined pitch}

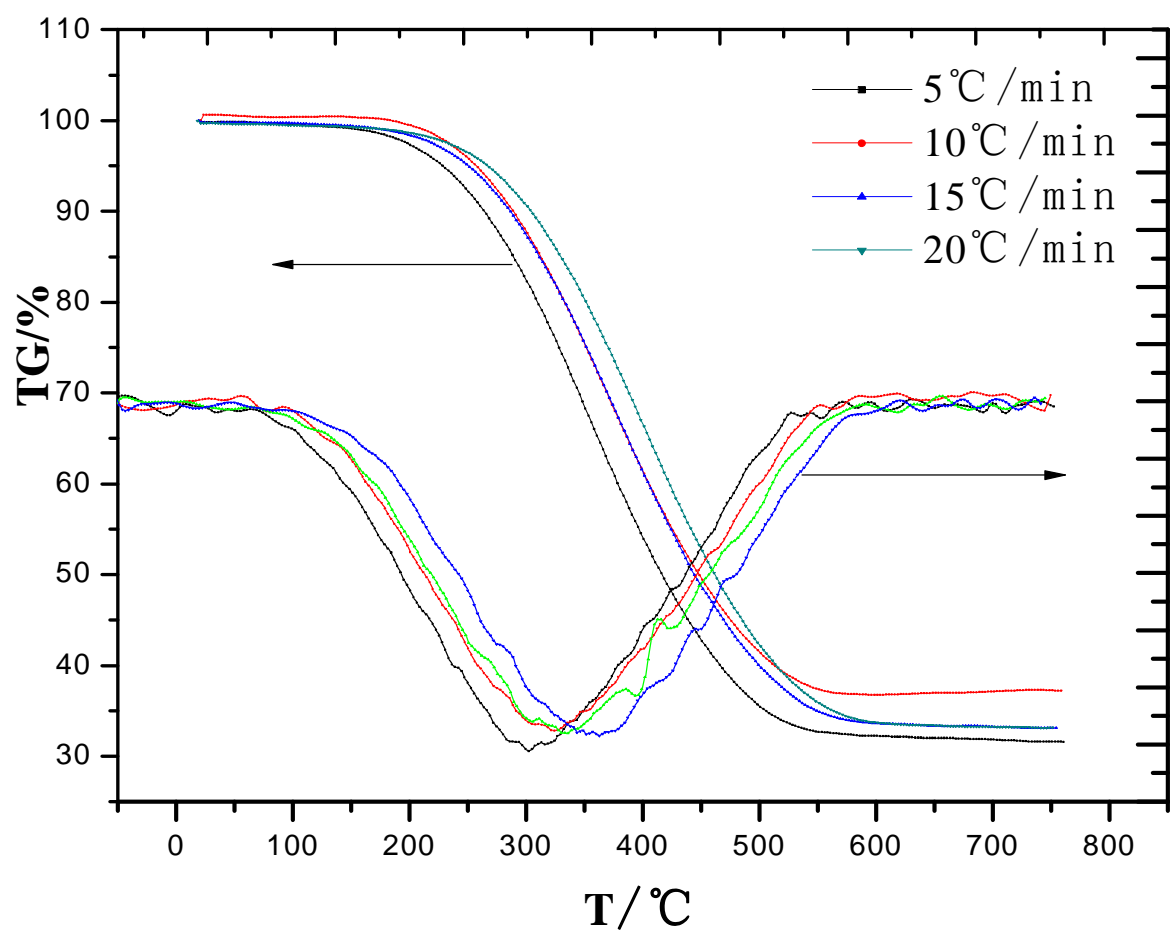

curves of CTP with different heating rate

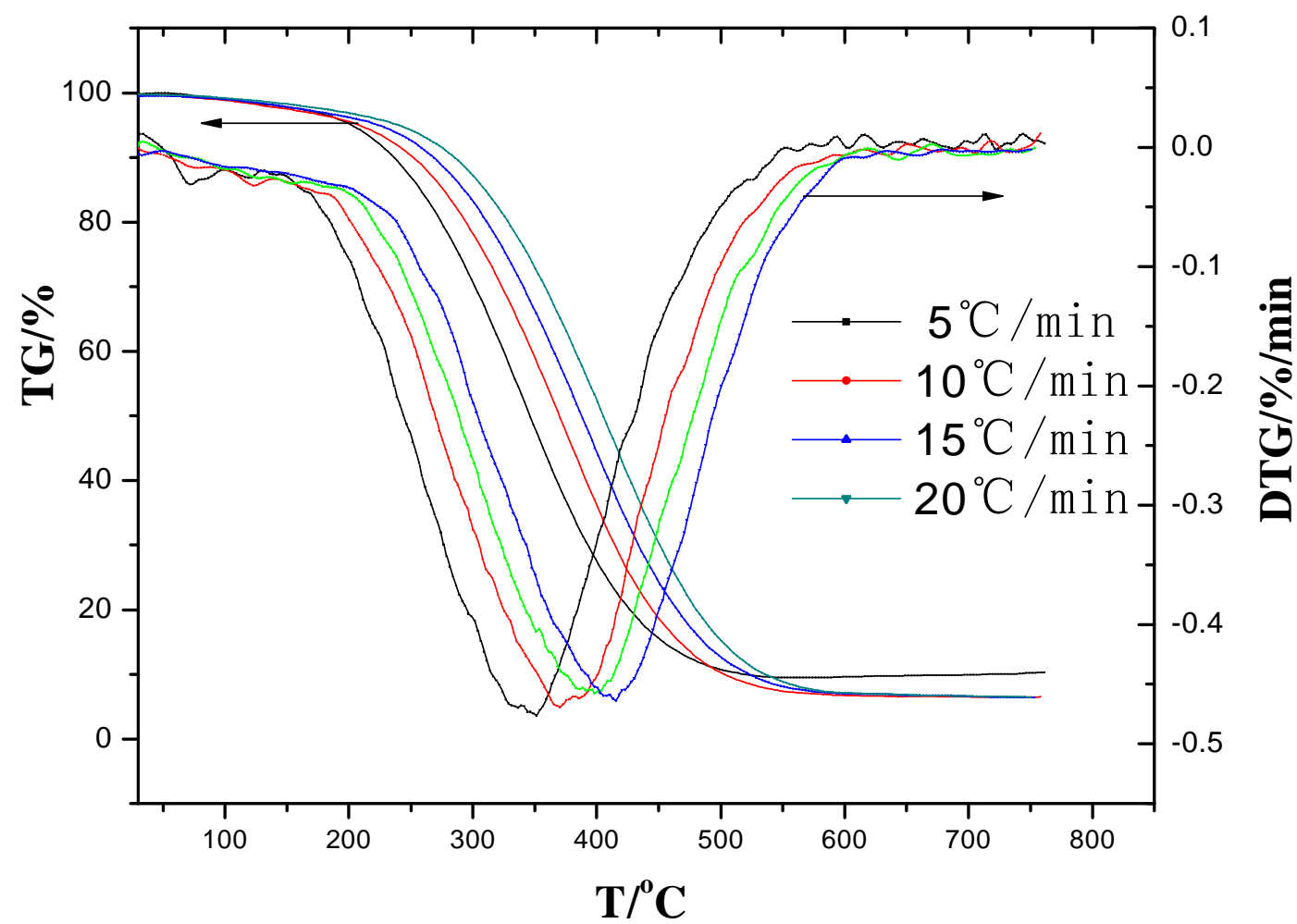

Fig.2 TG/DTG curves of the refined pitch with different heating rate

\section{Kissinger method}

Kissinger method is often used based on the DSC or DTA data to calculate kinetic parameters [6]. Assuming that the maximum reaction rate temperature is the maximum value of DTA or DSC peak curve. But in fact, the maximum reaction rate temperature is not exactly the peak maximum value of DTA or DSC. Therefore, the actual analysis often use Tp, the peak temperature of DTG curve 
instead of peak temperature of DSC curve temperature to correct, to make calculation. Kissinger equation:

$$
\ln (\beta / \mathrm{Tp} 2)=\ln (\mathrm{AR} / \mathrm{E})-(\mathrm{E} / \mathrm{RTp})
$$

In the formula, $\beta$ represents the temperature rising rate, $\mathrm{K} / \mathrm{min}$. $\mathrm{A}$ is pre-exponential factor, $\mathrm{E}$ is the apparent activation energy, $\mathrm{J} / \mathrm{mol}$. And $\mathrm{R}$ is the gas equilibrium constant. Fig. 1 is the curve of $\ln (\beta / \mathrm{Tp} 2)$ versus $1 / \mathrm{Tp}$. By the gradient of the straight line, the activation energy of CTP and the refined pitch can be worked out as $84.74 \mathrm{KJ} \cdot \mathrm{mol}^{-1} 79.30 \mathrm{KJ} \cdot \mathrm{mol}^{-1}$, respectively. The intercept, i.e. $\ln \mathrm{A}$, were 14.40 and 12.06 , respectively. The higher the activation energy $\mathrm{E}$, the more sensitive to the temperature, the temperature sensitivity of CTP is stronger than that of the refined pitch, and the reaction rate of CTP increases more than the refined pitch with the increase of temperature. The higher the pre-exponential factor A, the higher the reaction activity, CTP shows more reactive than the refined pitch. That is to say, the refined pitch is less temperature sensitivity than CTP, and the reaction activity increase is smaller with the increase of temperature. In the process of preparing the carbon materials, the lower temperature sensitivity makes the reaction be carried out at a slower speed, keeping the system at lower viscosity, which is beneficial to the mesophase formation and the processing of high performance carbon materials.

\section{Flynn-Wall-Ozawa method}

According to the TG/DTG curve of the two pitches at different heating rates, the Flynn-Wall -Ozawa method was used to calculate $\mathrm{E}[7,8]$ :

$$
\ln \beta=\ln [\mathrm{AE} / \mathrm{RG}(\alpha)]-5.3305-1.0516 \mathrm{E} / \mathrm{RT}
$$

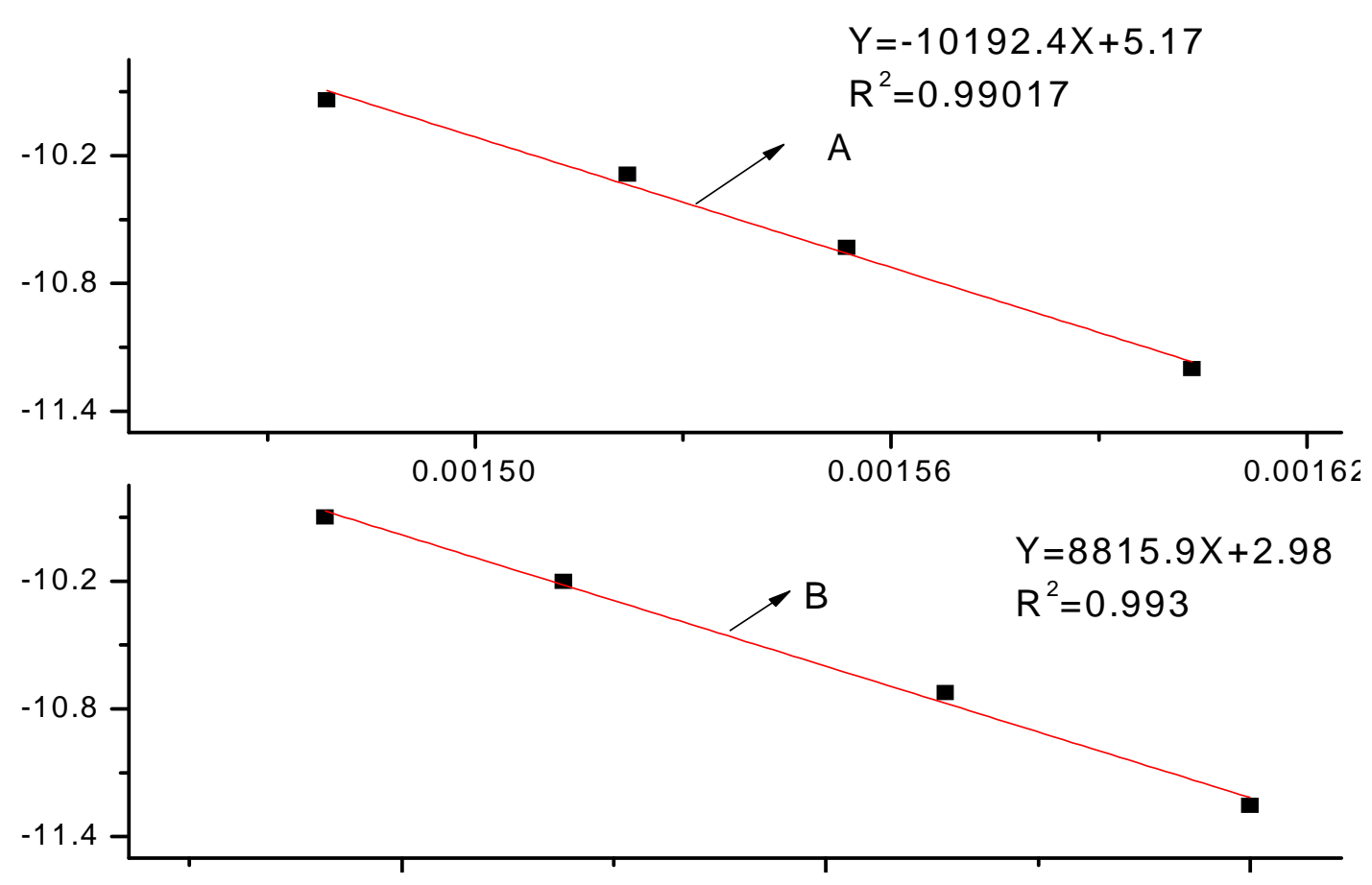

Fig.3. Kissinger formula fitting equation .

A: CTP; B: the refined pitch 


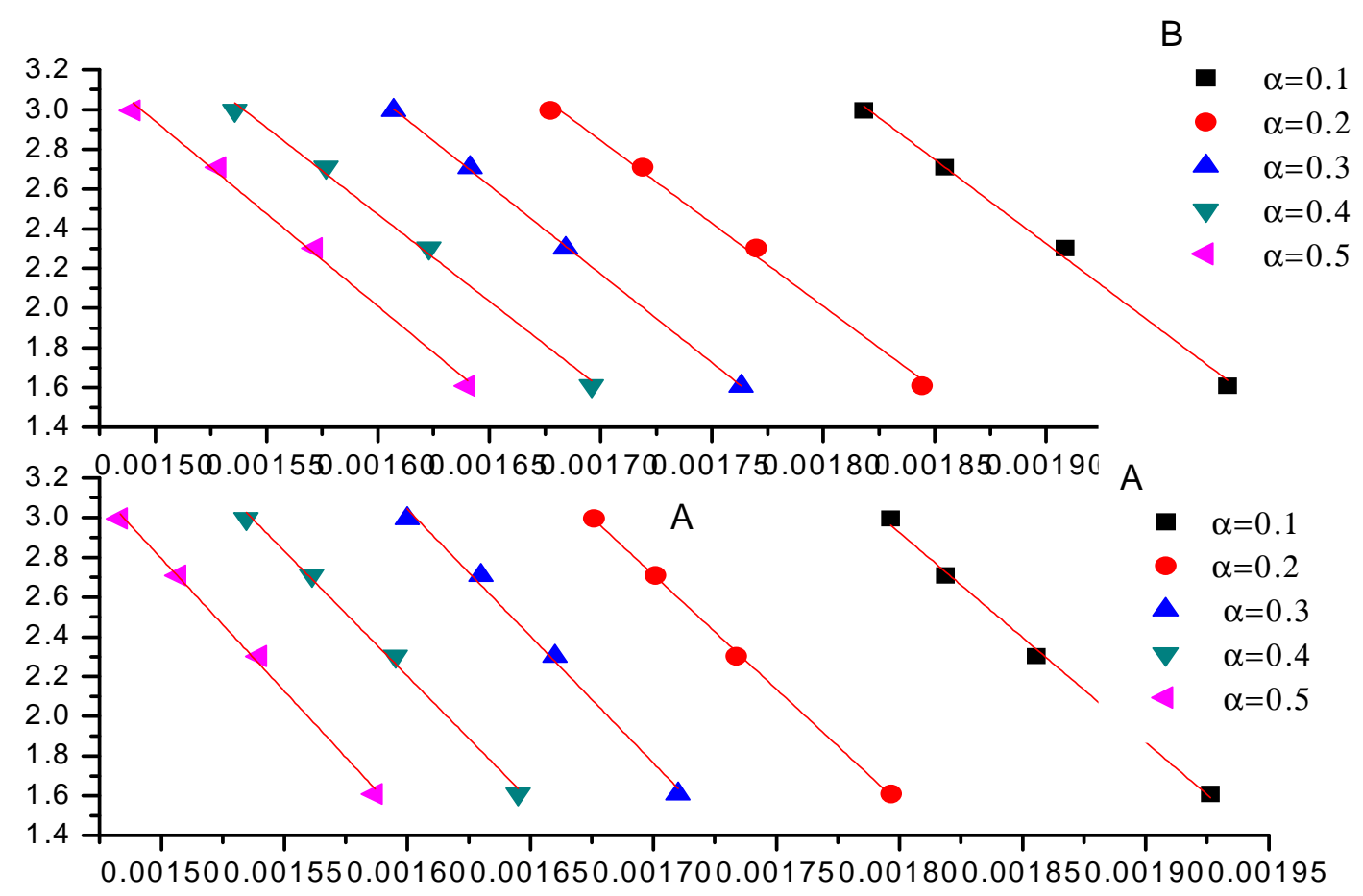

Fig. $4 . \ln \beta$ vs. $1 / \mathrm{T}$.

A: CTP; B:the refined pitch.

On the TG/DTG curve, $\mathrm{T}$ values at the same conversion rate are selected from the curve when different temperature elevating rate are taken. The value of $\ln \beta$ is plotted against the value of $1 / \mathrm{T}$ (Fig. 3), and the gradient of the line can be worked out $E$ value. The Value of $\ln \beta$ is plotted against 1/T. As is shown in Table 2, E of CTP and the refined pitch are $69.22 \mathrm{KJ} / \mathrm{mol}$ and $94.45 \mathrm{KJ} / \mathrm{mol}$, respectively. The results were similar to the results of 3.2.1., also suggesting that the temperature sensitivity of the CTP was stronger than that of the refined asphalt.

Table 2 Determination of E based on Flynn-Wall-Ozawa method

\begin{tabular}{|c|c|c|c|c|c|c|}
\hline \multirow[t]{4}{*}{ СТP } & $\alpha$ & 0.1 & 0.2 & 0.3 & 0.4 & 0.5 \\
\hline & $\mathrm{E}(\mathrm{KJ} / \mathrm{mol})$ & 83.43 & 91.05 & 92.61 & 99.55 & 105.62 \\
\hline & $\mathrm{R} 2$ & 0.9958 & 0.9990 & 0.9939 & 0.9949 & 0.9961 \\
\hline & Average $\mathrm{E}(\mathrm{KJ} / \mathrm{mol})$ & & & 94.45 & & \\
\hline \multirow{2}{*}{$\begin{array}{c}\text { Refined } \\
\text { pitch }\end{array}$} & $\mathrm{E}(\mathrm{KJ} / \mathrm{mol})$ & 66.78 & 65.96 & 70.60 & 69.12 & 73.65 \\
\hline & R2 & 0.9948 & 0.9938 & 0.9996 & 0.9946 & 0.9944 \\
\hline
\end{tabular}

\section{Reaction kinetics of pitch}

Thermal decomposition mechanism of pitch is speculated through plotting according to the thermal decomposition type which had been proposed. The apparent activation energy of the thermal decomposition is worked out based on the linear correlation of the curves and the given thermal decomposition kinetic equation. The apparent activation energy of three methods is compared with this value, and the dynamic expression is analyzed, then the thermal reaction mechanism is analysed according to the physical meaning of the expression. According to the kinetics equation of reaction $f(\alpha)=(1-\alpha)^{n}$, Arrhenius equation $k(t)=A \exp (-E / R T)$ and $\mathrm{d} \alpha / \mathrm{dt}=\beta \mathrm{d} \alpha / \mathrm{dT}=\mathrm{k}(\mathrm{T}) \cdot \mathrm{f}(\alpha)$, integral equation $\mathrm{g}(\alpha)$ of conversion is attained. The different reaction mechanism of expression $\mathrm{g}(\alpha)$ is calculated based on Coats-Redfern equation(equation (3)), The value of $\ln \left[\mathrm{g}(\alpha) / \mathrm{T}^{2}\right]$ is plotted against the value of $1 / \mathrm{T}$. The apparent activation energy of different reaction mechanisms can be obtained by the gradient of the curve and linear correlation. Thirty 
kinds of $g(\alpha)$ expression is listed in Table 3. The activation energy and the pre-exponential factor of these expressions must meet the following conditions: (1) $0<\mathrm{E}<400 \mathrm{KJ} / \mathrm{mol}$; (2) $\left|\left(\mathrm{E}_{0}-\mathrm{E}\right) / \mathrm{E}_{0}\right| \leq 0.3$, E0 is attained according to Flunn-Wall-Ozawa method; (3) $\left|\left(\ln \mathrm{A}-\ln \mathrm{A}_{0}\right) / \ln \mathrm{A}\right| \leq 0.3$, value of $\ln \mathrm{A} 0$ is calculated according to Kissinger method. In summary, ranges of CTP and the refined pitch are $66.12 \mathrm{KJ} / \mathrm{mol} \leq \mathrm{E} \leq 122.78 \mathrm{KJ} / \mathrm{mol}, \quad 11.08 \leq \ln \mathrm{A} \leq 20.57 \quad$ and $\quad 48.45 \mathrm{KJ} / \mathrm{mol} \quad \leq \mathrm{E} \leq 89.99 \mathrm{KJ} / \mathrm{mol}$, $9.28 \leq \ln \mathrm{A} \leq 17.23$, respectively. Taking $10 \mathrm{oC} / \mathrm{min}$ heating rate as an calculation example, the result is listed in Table 4, the reaction mechanism functions of CTP and the refined pitch both are $[-\ln (1-\alpha)] 2$. However, reaction rate of CTP is more sensitivity to temperature than that of the refined pitch. With the increase of temperature, the reaction rate of CTP is increased faster than the refined pitch. In other word, The reaction rate of the refined pitch is more mild with change of temperature and the reaction activity is low. In the process of preparation of high performance carbon materials, the carbonization reaction of the refined pitch is carried out at a slow speed, which makes the viscosity of the reaction system is low, Low viscosity is beneficial to mesophase formation and growth.

Table 3 The algebraic expression of $g(\alpha)$ for the various kinetic reaction models

\begin{tabular}{ll|ll}
\hline No. & $\mathrm{g}(\alpha)$ & No. & $\mathrm{g}(\alpha)$ \\
\hline 1 & $\alpha$ & 9 & $-\ln (1-\alpha)$ \\
2 & $\alpha+(1-\alpha) \ln (1-\alpha)$ & $10-16$ & {$[-\ln (1-\alpha)] \mathrm{n}, \mathrm{n}=2 / 3,1 / 2,1 / 3,4,1 / 4,2,3,4$} \\
3 & $(1-2 \alpha / 3)-(1-\alpha) 2 / 3$ & $17-22$ & $1-(1-\alpha) \mathrm{n}, \mathrm{n}=1 / 4,1 / 3,1 / 2,2,3,4$ \\
$4-5$ & {$[1-(1-\alpha) 1 / 3] \mathrm{n},(\mathrm{n}=2,0.5)$} & $23-27$ & $\alpha \mathrm{n}, \mathrm{n}=1 / 4,1 / 3,1 / 2,1,3 / 2$ \\
6 & {$[1-(1-\alpha) 1 / 2] 1 / 2$} & 28 & $(1-\alpha)-2$ \\
7 & {$[(1-\alpha) 1 / 3-1] 2$} & 29 & $(1-\alpha)-1-1$ \\
8 & {$[(1 /(1+\alpha) 1 / 3-1] 2$} & 30 & $(1-\alpha)-1 / 2$ \\
\hline
\end{tabular}


Table 4. Results of 30 types of kinetic equations calculated with Coats-Redfern method under

\begin{tabular}{llll|lll} 
& \multicolumn{5}{c}{$\beta=10 \mathrm{~K} / \mathrm{min}$} \\
$\mathrm{No}$ & $\mathrm{CTP}$ & & & \multicolumn{2}{l}{ The refined pitch } & \\
1 & $\mathrm{E} /(\mathrm{KJ} / \mathrm{mol})$ & $\ln \mathrm{A}$ & $\mathrm{R} 2$ & $\mathrm{E} /(\mathrm{KJ} / \mathrm{mol})$ & $\mathrm{ln} \mathrm{A}$ & $\mathrm{R} 2$ \\
2 & 61.47 & 8.15 & 0.97299 & 61.77 & 8.51 & 0.9913 \\
3 & 67.25 & 8.84 & 0.98355 & 67.95 & 9.31 & 0.9965 \\
4 & 70.84 & 8.84 & 0.98335 & 70.36 & 8.39 & 0.9979 \\
5 & 76.09 & 9.35 & 0.99174 & 75.12 & 9.52 & 0.9995 \\
6 & 11.21 & -2.17 & 0.96773 & 11.27 & -2.06 & 0.9949 \\
7 & 10.16 & -2.3 & 0.95323 & 10.29 & -2.17 & 0.9996 \\
8 & 75.99 & 9.32 & 0.99218 & 75.23 & 9.05 & 0.9995 \\
9 & 48.82 & 2.80 & 0.94953 & 49.11 & 3.13 & 0.9786 \\
10 & 37.28 & 4.29 & 0.99572 & 36.38 & 4.30 & 0.9998 \\
11 & 21.21 & 0.88 & 0.99346 & 20.90 & 0.93 & 0.9999 \\
12 & 13.35 & -0.98 & 0.98936 & 13.17 & -0.93 & 0.9999 \\
13 & 5.39 & -3.30 & 0.96267 & 5.39 & -3.24 & 0.9952 \\
14 & 179.65 & 31.13 & 0.99787 & 175.36 & 31.09 & 0.9999 \\
15 & 1.47 & -5.30 & 0.73114 & 1.56 & -5.17 & 0.9559 \\
16 & 84.47 & 13.48 & 0.99734 & 82.27 & 13.44 & 0.9995 \\
17 & 131.01 & 22.18 & 0.99743 & 129.45 & 23.39 & 0.9997 \\
18 & 34.13 & 2.13 & 0.99054 & 33.46 & 2.17 & 0.9996 \\
19 & 32.60 & 2.06 & 0.98791 & 32.544 & 2.22 & 0.9990 \\
20 & 30.75 & 2.00 & 0.98255 & 30.78 & 2.18 & 0.9946 \\
21 & 16.35 & -0.41 & 0.84905 & 17.61 & 19.91 & 0.9206 \\
22 & 10.03 & -1.94 & 0.64105 & 12.515 & -1.13 & 0.8214 \\
23 & 5.57 & -3.26 & 0.3789 & 7.34 & -2.58 & 0.5872 \\
24 & 1.42 & -5.97 & 0.42833 & 1.08 & -6.14 & 0.4839 \\
25 & 1.57 & -5.36 & 0.36761 & 1.903 & -5.07 & 0.6672 \\
26 & 7.69 & -2.77 & 0.88089 & 7.99 & 20.94 & 0.9553 \\
27 & 43.99 & 4.95 & 0.9687 & 44.146 & 5.21 & 0.9904 \\
28 & 41.59 & 7.26 & 0.87781 & 37.745 & 6.61 & 0.8298 \\
29 & 52.09 & 7.83 & 0.99733 & 50.067 & 7.66 & 0.9868 \\
30 & 2.79 & -3.89 & 0.37505 & 1.501 & 4.72 & 0.1212 \\
\hline & & & & & &
\end{tabular}

\section{Conclusion}

Reaction kinetics of CTP and the refined pitch is studied using the method of synchronous thermal analysis. The thermal decomposition process of CTP can be divided into three stages, which correspond to different physical and chemical changes. $200-550^{\circ} \mathrm{C}$ is the main stage of CTP pyrolysis. Different heating rate has different effect on carbonization.

The apparent activation energy E of CTP and the refined pitch is about $84.74 \mathrm{KJ} \cdot \mathrm{mol}^{-1}$ and 79.30 $\mathrm{KJ} \cdot \mathrm{mol}^{-1}$ based on Kissinger method, While $94.45 \mathrm{KJ} \cdot \mathrm{mol}^{-1}$ and $69.22 \mathrm{KJ} \cdot \mathrm{mol}^{-1}$ based on Flynn-Wall-Ozawa method, respectively. The pre-exponential factor $\ln$ A of CTP and the refined pitch is 14.40 and 12.06, respectively. The reaction mechanism function of CTP and the refined pitch is $[-\ln (1-\alpha)]$ according to Coats-Redfern integral method. 
According to calculation results, CTP shows more sensitivity to temperature change and stranger reaction activity.

Acknowledgement:

This research was financially supported by the "Petrochina Innovation Foundation" (No. 2015D-5006-0408) and "Excellent Youth Development Foundation of Zhengzhou ".

\section{Reference}

[1] Gao Jinsheng, Zhang Dexiang, Wan Chen. The development and proposal of coal tar processing technology [J]. Coal Chemical Industry. 1(1999): 3-6.

[2] Egashira M, Hoshii K, Araki T,et al. Effects of fullerene addition on the carbonization of synthetic naphthalene[J]. Carbon, 36(2)(1998): 1739-1747.

[3] Youliang Cheng, Changqing Fang, Jian Su, Ruien Yu, Tiehu Li. Carbonization behavior and mesophase conversion kinetics of coal tar pitch using a low temperature molten salt method. Journal of Analytical and Applied Pyrolysis. 109(2014): 90-97.

[4] Hengfu Shui, Yingtong Feng, Benxian Shen, Jinsheng Gao. Kinetics of mesophase transformation of coal tar pitch. Fuel Processing Technology 55(1998): 153-160.

[5] Andrzej Mianowski, Stanislaw Blazewicz, Zbigniew Robak. A nalysis of the carbonization and formation of coal tar pitch mesophase under dynamic conditions. Carbon 41(2003): 2413-2424.

[6] Kissinger H. E. Reaction kinetics in differential thermal analysis[J]. Analytical Chemistry, 29(11)(1957): 1702-1706.

[7] T. Ozawa. A new method of analyzing thermo gravimetric data [J]. Bulletin of the Chemical Society of Japan, 38(11)(1965): 1881-1886.

[8] Flynn J. H., Wall L. A. A quick direct method for the determination of activation energy from thermogravimetric data [J]. Journal of Polymer Science, Part B:Polymer Physical, 4(5)(1966): 323-328.

[9]A. W. Coats and J. P. Redfern, Kinetic Parameters from Thermogravimetric Data, Nature, Vol. 201(1964), pp.68-69.

[10]C. Y. Ou, C. H. Zhang, S. D. Li, L. Yang and J. J. Dong, Thermal Degradation Kinetics of Chitosan-Cobalt Complex as Studied by Thermogravimetric Analysis, Carbohydrate Polymers, Vol. 82, No. 4(2010) , pp. 1284-1289. 